\title{
Visual detection of 2,4,6-trinitrotolune by molecularly imprinted colloidal array photonic crystal
}

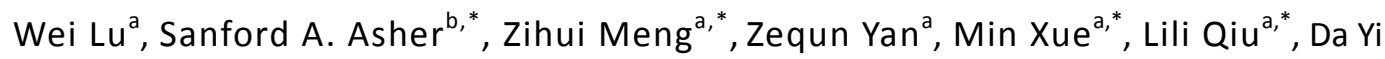

${ }^{a}$ Beijing Institute of Technology, Beijing, 100081, P. R. China

${ }^{b}$ Department of Chemistry, University of Pittsburgh, Pittsburgh, PA 15260, U. S.

\section{ABSTRACT}

We developed a photonic crystal (PhC) sensor for the quantification of 2,4,6trinitrotoluene (TNT) in solution. Monodisperse (210 $\mathrm{nm}$ in diameter) molecularly imprinted colloidal particles (MICS) for TNT were prepared by the emulsion polymerization of methyl methacrylate and acrylamide in the presence of TNT as a template. The MICs were then self-assembled into close-packed opal PhC films. The adsorption capacity of the MICs for TNT was $64 \mathrm{mg} \mathrm{TNT} / \mathrm{g}$. The diffraction from the PhC depended on the TNT concentration in a methanol/water (3/2, v/v) potassium dihydrogen phosphate buffer solution $(\mathrm{pH}=7.0,30 \mathrm{mM})$. The limit of detection (LOD) of the sensor is $1.03 \mu \mathrm{g}$. The color of the molecularly imprinted colloidal array (MICA) changed from green to red with an $84 \mathrm{~nm}$ diffraction red shift when the TNT concentration increased to $20 \mathrm{mM}$. The sensor response time was $3 \mathrm{~min}$. The PhC sensor was selective for TNT compared to similar compounds such as 2,4,6trinitrophenol, 2,4-dinitrotoluene, 2,6-dinitrotoluene, 2-nitromesitylene, 4nitrotoluene, 2-nitrotoluene, 1,3-dinitrobenzene, methylbenzene, 4-nitrophenol, 2nitroaniline, 3-aminophenol and 3-nitroaniline. The sensor showed high stability with little response change after three years storage. This sensor technology might be useful for the visual determination of TNT.

Keywords:

Photonic crystal

Molecular imprinting technology

2,4,6-trinitrotoluene

TNT visual detection

Chemical sensor

*Corresponding author at: Department of Chemistry, University of Pittsburgh, Pittsburgh, PA 15260, U. S. Phone: 412-624-8570, Fax: 412-624-0588. E-mail: asher@pitt.edu (S. Asher); School of Chemical Engineering and Engineering and Environment, Beijing Institute of Technology, Beijing, China, Phone: (8610)68913065. Fax: (8610)68913065. E-mail: $m$ zihui@yahoo.com (Z. Meng). E-mail: minxue@bit.edu.cn (M. Xue). E-mail: qiulili@bit.edu.cn (L. Qiu). 


\section{Introduction}

Detection of explosives such as TNT is important to prevent incidents of terrorism [1]. In addition, detection of TNT is important because it's a carcinogen that is often found near military installations [2]. In the work here we describe the development of molecularly imprinted photonic crystal (PhC) sensors for TNT detection. These PhC sensors utilize the diffraction from periodic arrays of particles to determine analyte concentrations. This enables the visual determination of the concentrations of specific chemical and biochemical species [3-12]. A somewhat related approach was recently taken by Fang et al., who utilized an inverse opal $\mathrm{PhC}$ sensor that used a hybrid monolayer of amino ligands and dye molecules to detect TNT vapor [13]. This sensor, which used fluorescence energy transfer is likely to be of less general utility.

Due to the high mechanical strength and flexibility, molecularly imprinted polymers (MIPs) were recently utilized to fabricate highly sensitive, selective and cost-effective molecular recognition materials [14-17]. Referred to as antibody mimics, the artificial materials contain thousands to millions of target-shaped cavities, which could bind specific molecules [18-21] and reduce the cross-absorptivity of the targets' analogues. And the state of MIPs have been used to enable TNT detection through the use of fluorescence [22, 23], quartz crystal microbalance (QCM) [24, 25], and surface plasma resonance measurements [26]. These sensors show high selectivity and sensitivity with a real-time and on-site detection result. However, the high technical knowledge requirements and harsh detection environments of these approaches still limit their utilization. By utilizing MIP polymers in $\mathrm{PhC}$, we can develop selective detection devices to visually detect analytes such as TNT $[14,27]$. We have been inspired in this work by recent advances in PhC sensors [28-30].

We recently developed a MIPs for selective TNT adsorption in real samples [31] and a molecularly imprinted colloidal array (MICA) for the sensing of endocrine disruptor compounds [32] by the self-assembly of molecularly imprinted colloidal particles (MICs). We are continuing the development of this material and this sensing technique by combining them to detect TNT. We described here a TNT sensor that can be used to visually estimate TNT concentrations in solution by the color of light diffracted from the $\mathrm{PhC}$ array sensor. 


\section{Experimental}

\subsection{Chemicals and materials}

Acrylamide (AM) and TNT were purchased from Kermel Reagents Development Center. 2,4-Dinitrotoluene (2,4-DNT), 2,6-dinitrotoluene (2,6-DNT), 2-nitromesitylene, 4-nitrotoluene (PNT), 2-nitrotoluene (ONT), 1,3-dinitrobenzene, methylbenzene, 4nitrophenol, 2-nitroaniline, 3-aminophenol and 3-nitroaniline were purchased from Sinopharm. 2,4,6-Trinitrophenol (TNP) was purchased from Weiss Experiment Product Co. Ltd, China. Methyl methacrylate (MMA) was purchased from Bodi Chemical. Potassium persulfate (KPS) was purchased from Xilong Chemical. Potassium dihydrogen phosphate (KDP) and potassium hydroxide $(\mathrm{KOH})$ were purchased from Beijing Chemical Plants and Feng Chuan Chemical Reagent Science and Technology Ltd, respectively. All of above chemicals were of analytical grade. Methanol and acetic acid were of chromatographic grade and were purchased from Yu Wang Industrial Co. Ltd. Beijing Chemical Plant provided the sulfuric acid $\left(\mathrm{H}_{2} \mathrm{SO}_{4}, 98 \%\right)$ and hydrogen peroxide $\left(\mathrm{H}_{2} \mathrm{O}_{2}, 30 \%\right)$ solutions. Deionized water was produced by an ultrapure water purifier manufactured by Aquapro (Chongqing, China).

The centrifuge tubes, glass tubes and slides $(50 \times 24 \times 0.12 \mathrm{~mm})$ were purchased from the Weiss Experiment Product Co. Ltd, China. The adhesive tape was purchased from Deli Tape Co. Ltd. The nylon membranes with $0.22 \mu \mathrm{m}$ diameter pores were provided by Agela Technologies.

\subsection{Preparation of the MICs}

The MICs were prepared by emulsion polymerization [32,33]. Typically $0.17 \mathrm{mmol}$ of the template molecule (TNT) and $1 \mathrm{mmol}$ AM were dissolved in $7 \mathrm{~mL}$ of MMA in a 20 $\mathrm{mL}$ disposable scintillation vial. The mixture was sonicated for $20 \mathrm{~min}$ and then diluted with $85 \mathrm{~mL}$ water in a four neck flask $(250 \mathrm{~mL})$. The mixture was deoxygenated by bubbling with nitrogen at $75^{\circ} \mathrm{C}$. The polymerization was initiated by addition of $5 \mathrm{~mL}$ of KPS $(0.74 \mathrm{mmol})$ buffer. The obtained mixture was stirred at $80{ }^{\circ} \mathrm{C}$ for $45 \mathrm{~min}$.

The resulting MICs were centrifuged and washed with a methanol/acetic acid (8/2, $v / v)$ solution until the TNT was undetectable by high performance liquid 
chromatography (HPLC). The particles were then washed four times with $15 \mathrm{~mL}$ methanol, and then water for 40 min each including 3 min of sonication.

Non-imprinted colloidal particles (NICs) were synthesized in the same manner but in the absence of TNT. Polymethyl methacrylate (PMMA) particles were also prepared in the same manner in the absence of TNT and AM comonomer.

\subsection{Fabrication of MICA films}

The MICA fabrication method is shown in Figure 1. The glass slides were cleaned and made hydrophilic by immersing them into a $\mathrm{H}_{2} \mathrm{SO}_{4} / \mathrm{H}_{2} \mathrm{O}_{2}(7 / 3, \mathrm{v} / \mathrm{v})$ solution and then

rinsing them with water. The monodisperse MICs were dispersed in deionized water (0.25 wt\%) and then stored in square glass containers in which the glass slides were immersed vertically. The MICs dispersion was evaporated at $30^{\circ} \mathrm{C}$ for two days while exposed to a relative humidity of $50 \%$. As the dispersion evaporated, the monodisperse MICs deposited three-dimensional (3D) close-packed MICA films on both sides of the glass slides. These MICA films attached to the slides were then pressed against the adhesive tape. The tape was removed such that the MICA peeled off the glass slides.

\subsection{Characterization of MICs and MICA.}

To investigate the response of the MICs to TNT, we prepared $1 \mathrm{~mL}$ methanol/water $(3 / 2, v / v)$ solutions at TNT concentrations between $2.5-20 \mathrm{mM}$. We then incubated 10 $\mathrm{mg}$ of MICs in $1 \mathrm{~mL}$ TNT solutions in $1.5 \mathrm{~mL}$ centrifuge tubes for $40 \mathrm{~min}$. These dispersions were then centrifuged and the supernatant was collected and filtered through $0.22 \mu \mathrm{m}$ pore diameter nylon membranes prior to HPLC measurements. We used HPLC to determine the residual TNT remaining in the incubation solutions. The results were used to calculate the amount of TNT adsorbed by the MICs and NICs.

A Quanta ${ }^{\mathrm{TM}} 250$ scanning electron microscope (SEM) from FEI was utilized to obtain images by collecting secondary electrons with an Everhart-Thornley detector. We used a beam voltage of 50-20 kV with a working distance of $10 \mathrm{~mm}$. 


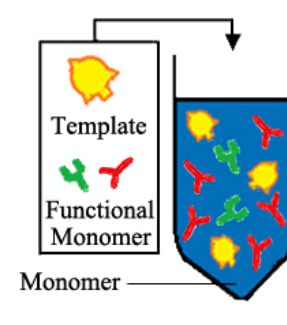

Dissolution

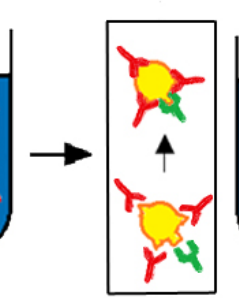

Prepolymerization

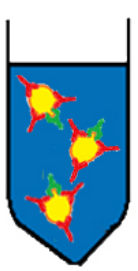

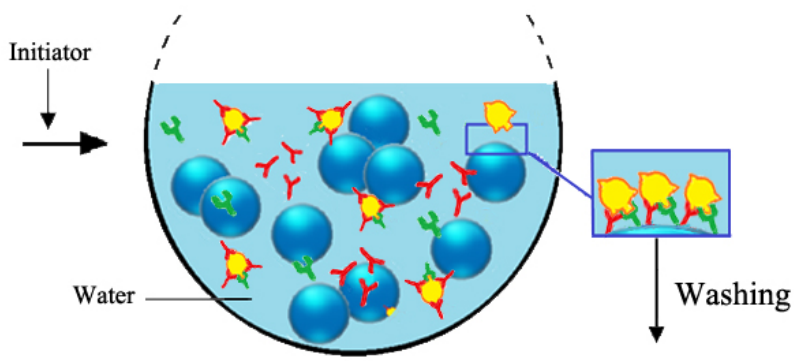

Emulsion Polymerization sriss
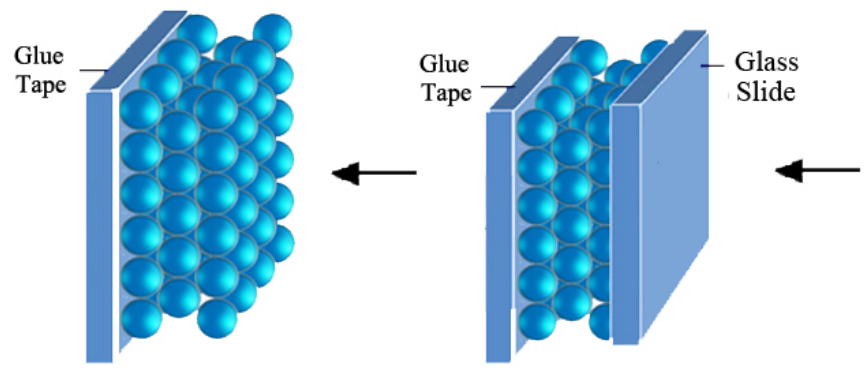

Solidification

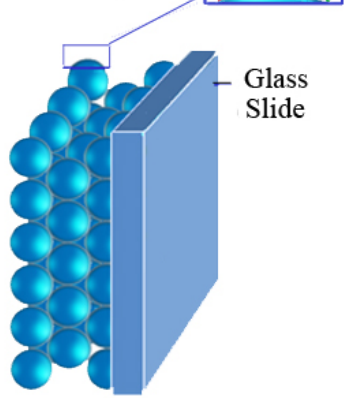

Self-assembly

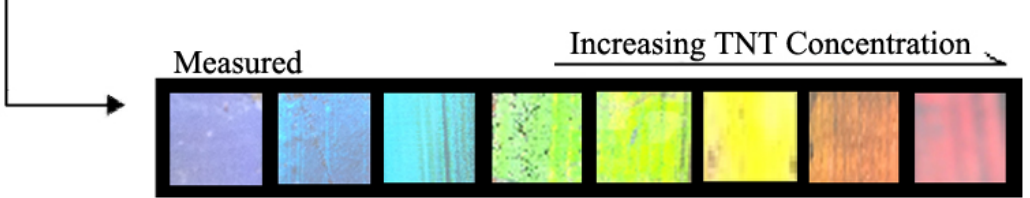

Color of MICA

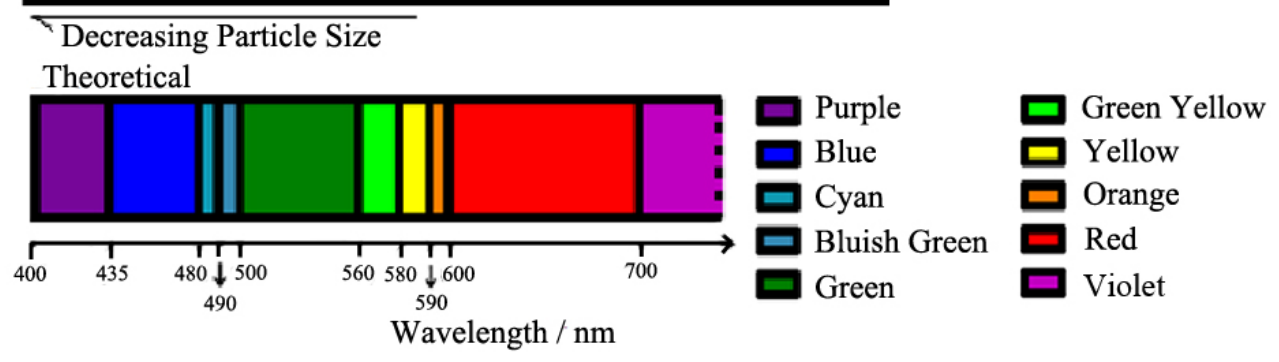

Fig. 1. Fabrication of MICA. Self-assembled MICs array was transferred from the glass slide to the tape. Also shown are the MICA diffraction colors.

Particle sizes of MICs were measured with a Dynamic Light Scattering (DLS) instrument from Brookhaven Instruments Corporation. HPLC measurements were performed by using a Shimadzu LC-20AT HPLC instrument, with a detection wavelength of $235 \mathrm{~nm}$. A Pronto SIL 100-5-C18 HPLC column (4.6 x $250 \mathrm{~mm}$, B\&W) was used with a methanol/water $(75 / 25, \mathrm{v} / \mathrm{v})$ mobile phase with a flow rate of $1 \mathrm{~mL} / \mathrm{min}$.

The diffraction spectrum from the MICA was recorded using an Aventes 2048TEC fiber 
optic spectrometer with an Avalight-DH-S-BAL light source and a FC-UV600-2-SR fiber optic reflection probe. The films were cut into $10 \times 5 \mathrm{~mm}$ pieces and equilibrated in a methanol/water $(3 / 2, \mathrm{v} / \mathrm{v})$ solution before measurements. The diffraction color changes of the films were recorded by a digital camera at a constant angle.

\section{Results and discussion}

\subsection{MICS TNT binding}

As discussed above we determined TNT binding by incubating $10 \mathrm{mg}$ MICs for $40 \mathrm{~min}$ in $1 \mathrm{~mL}$ TNT methanol/water solutions (3/2, v/v). Subsequent HPLC measurements determined the TNT concentrations remaining after incubation. The linear range was 0.3-30 mM (Fig. S-1) with the limit of detection (LOD) of $3 \times 10^{-4} \mathrm{mM}(\mathrm{S} / \mathrm{N}=3)$. Figure 2A shows that the MICs absorbed $44.9 \mathrm{mg}$ TNT /g upon equilibrium in $1 \mathrm{~mL} 3.2 \mathrm{mM}$ TNT solution. The MICs absorbed 2 more fold TNT than that did the NICs in an $8 \mathrm{mM}$ TNT solution upon equilibrium.

Figure 2B shows that for an initial TNT concentration of $15 \mathrm{mM}$, absorption saturation occurred within $1 \mathrm{hr}$ with twice as much as TNT absorbed by the MICs compared to the NICs. The final residual TNT concentrations were found to be $11.8 \mathrm{mM}$ and $13.7 \mathrm{mM}$ after absorption of MICs and NICs reached equilibrium, respectively.
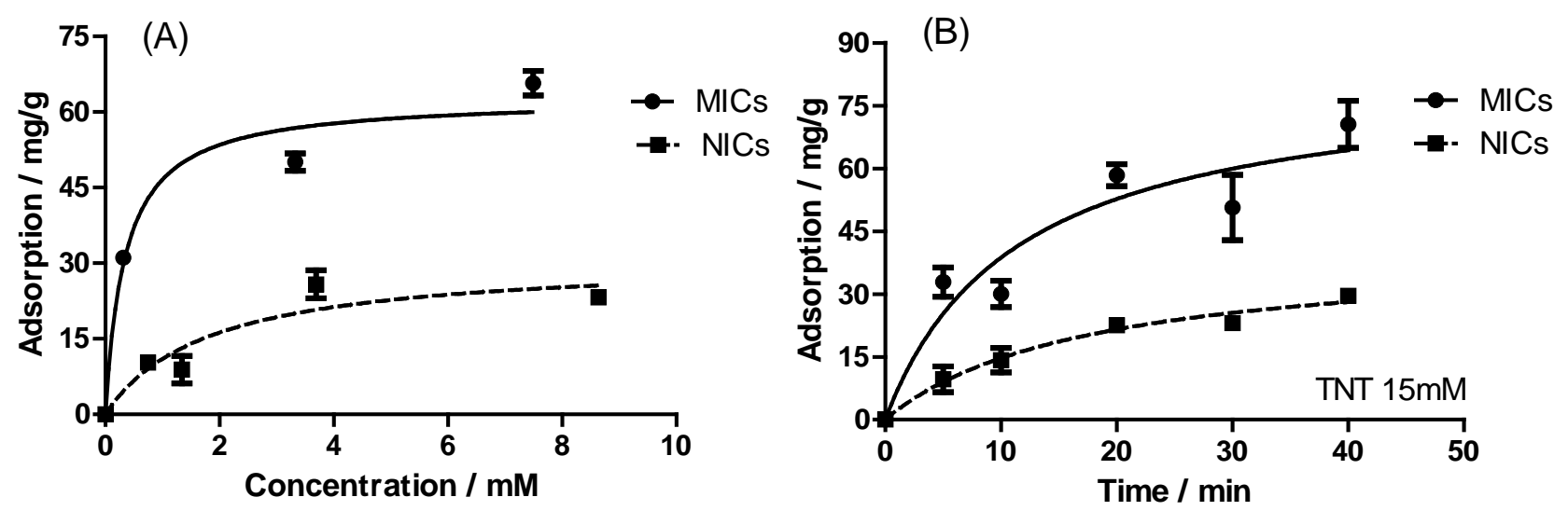

Fig. 2. (A) Adsorption isotherms of TNT on MICs and NICs. (B)Time dependence of TNT adsorption (mg/g of particles) for MICs and NICs from $15 \mathrm{mM}$ TNT dissolved in $1 \mathrm{~mL}$ methanol/water (3/2, v/v) solutions.

The higher TNT absorption of MICs compared to NICS, derived from the imprinting 
[21, 34]. By adopting the single site binding model [35], we can calculate the adsorption capacity and the equilibrium binding constant, $\mathrm{K}_{\mathrm{a}}$ from the binding isotherms.

TNT binds not only to the imprinted cavities, but also binds with lower affinity to the sites containing amide groups distributed on the colloidal particles. By using a one site binding model, the resulting adsorption capacity was determined to be $63.6 \mathrm{mg} / \mathrm{g}$ and $31.8 \mathrm{mg} / \mathrm{g}$ for the MICs and NICs respectively. Thus, the MICs affinity is twice that of NICs. The equilibrium binding constant $K_{a}$ was determined to be $2.88 \mathrm{~m} \mathrm{M}^{-1}$ and 0.55 $\mathrm{mM}^{-1}$ for the MICs and NICs respectively. If we define an imprinting efficiency parameter (I) as the ratio of equilibrium binding constants, $\mathrm{I}=\mathrm{K}_{\mathrm{a}}{ }^{\mathrm{MICs}} / \mathrm{K}_{\mathrm{a}}{ }^{\mathrm{NICS}}=5.0$.

\subsection{Fabrication and characterization of the MICA}

The MICs prepared by emulsion polymerization were monodisperse as shown in Figure $3 \mathrm{~A}$. The MICs self-assembled on the surface of glass slides to form 3D MICA films as the solvent evaporates. We then transferred the MICA films from the glass slides to the adhesive tape. The resulting 3D MICA on the tape was not close-packed due to the mechanical strains occurred during the peeling off of the array from the glass slide. We then observed a $\sim 10 \mathrm{~nm}$ surface to surface particle spacing of the MICA (Fig 3B).
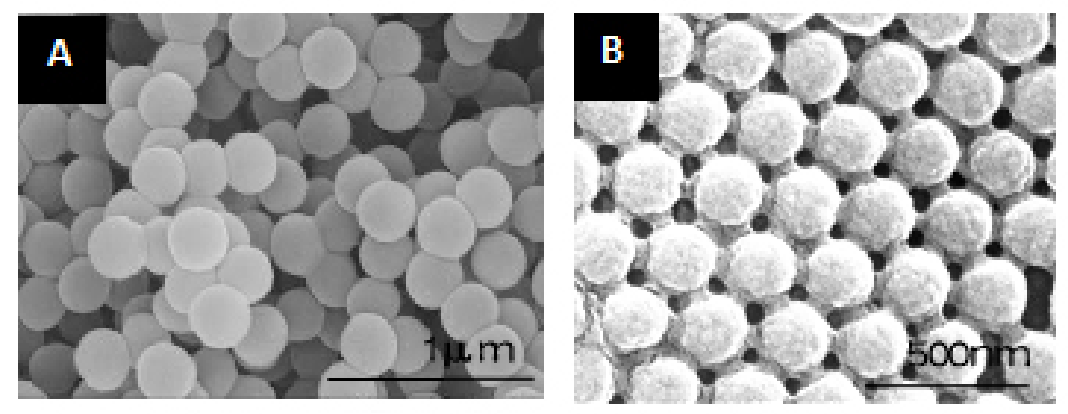

Fig. 3. SEM images of (A) TNT MICs aggregates, (B) MICA films

The periodic structure of the MICA diffracts light according to Bragg's law. For an ordered array, the diffracted wavelength $\lambda_{\max }$ follows: 


$$
\lambda_{\max }=1.633(d / m)\left(D / D_{0}\right)\left(n_{\mathrm{a}}{ }^{2}-\sin ^{2} \theta\right)^{1 / 2}
$$

Where $d$ is the diameter of the colloidal particles; $m=1$ is the order of Bragg diffraction; $n_{a}$ is the average refractive index of the PhC; $\mathrm{D} / \mathrm{D}_{0}$ is the swelling ratio of the MICs; $\theta$ is the angle between the incident light and the normal to the crystal surface. $n_{a}$ can be calculated using equation (2):

$$
\mathrm{n}_{\mathrm{a}}=\left[\mathrm{n}_{1}^{2} f+\mathrm{n}_{2}^{2}(1-f)\right]^{1 / 2}
$$

When the MICA film is dry, $n_{a}$ is the average refractive indexes of PMMA colloidal particles $\left(n_{1}=1.48\right)$ and air $\left(n_{2}=1.00\right)$ [36]. We assumed a close-packed array with a volume fraction, $f$, of 0.74 . If the incident light $\theta=90^{\circ}, \lambda_{\max }$ depends only on $d$. If $\lambda_{\max }$ occurs in the visible spectral region, the photonic crystal will appear brightly colored.

To experimentally determine the dependence of the diffraction wavelength on the particle diameter, we examined the diffraction of MICAs with particles of different diameters. The relationship of the particle diameter, the diffraction wavelength and the visually evident color is shown in Table 1.

\section{Table 1}

Comparison of the particle diameter, diffraction wavelength and the perceived MICA color

\begin{tabular}{ccc}
\hline Particle Diameter/nm & Diffraction Wavelength/nm & Color \\
\hline 184 & 432 & Blue \\
191 & 466 & Green \\
209 & 507 & Green \\
220 & 539 & Yellow \\
243 & 575 & Red \\
260 & 687 & Violet
\end{tabular}

*the particle diameter and diffraction wavelength was characterized by SEM and fiber optic spectrometer, respectively.

We compared value of $\lambda_{\max }$ calculated from equation (1) with its measured value for the close-packed array. The factor, $D / D_{0}$, was fixed as 1 when the film is dry. As shown in Figure 4 , the measured $\lambda_{\max }$ was slightly larger than the calculated. This probably resulted from the nonclose-packed expansion of the lattice constant, which caused by 
the mechanical strains during the peeling off of the array from the glass slide.

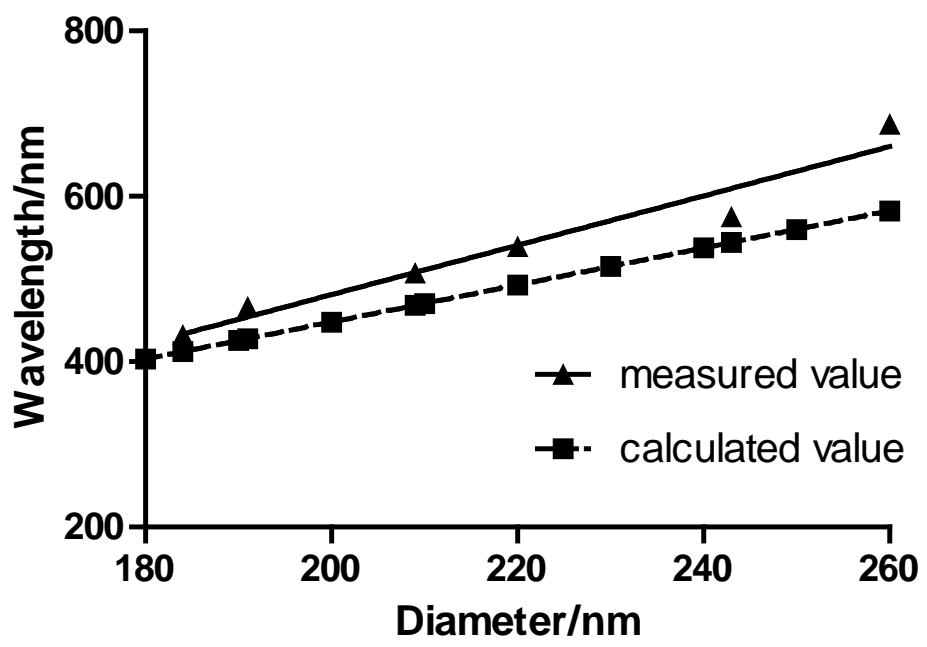

Fig. 4. Calculated and measured values of $\lambda_{\max }$ for different particles diameters.

\subsection{MICA detection of TNT}

The dependence of diffraction of the MICA films $(10 \times 5 \mathrm{~mm})$ in response to $5 \mathrm{mM}$ TNT solutions under different $\mathrm{pH}$, temperature and ionic strength solutions was monitored in $10 \mathrm{~mL}$ solutions using a fiber optic spectrometer. For these measurements, the MICA films were removed from solution after the TNT adsorption saturated. The films were then gently blotted dry. The diffraction of $180^{\circ}$ back diffracted light was measured, and the color changes were observed within 3 min. We observed that the diffraction red shifted $37 \mathrm{~nm}$ in response to a $5 \mathrm{mM}$ TNT methanol/water $(3 / 2, v / v)$ solution, while it red shifted $45 \mathrm{~nm}$ in response to a methanol/water solution that contained KDP (3/2, v/v, $10 \mathrm{mM} \mathrm{KDP} \mathrm{buffer,} \mathrm{pH}$ 7.0).

Thus, the diffraction wavelength also depended on the KDP concentration. For $5 \mathrm{mM}$ TNT the diffraction was further increased by $\sim 15 \mathrm{~nm}$ when the KDP concentration was increased to $30 \mathrm{mM}$. SEM measurements show that the particle diameter increased $\sim 5$ $\mathrm{nm}$ upon immersion in $30 \mathrm{mM}$ KDP buffer in the absence of TNT. Thus, KDP induced particle swelling may be partially responsible for the increased red shift.

To investigate the response of the MICA to 0-20 mM TNT solution concentrations, we incubated MICA and non-imprinted colloidal array (NICA) films (10 x 5mm pieces) in 10 
$\mathrm{mL}$ TNT methanol/water solutions (3/2, v/v, $30 \mathrm{mM}$ KDP buffer, $\mathrm{pH} 7.0)$. Figure 5 shows the MICA and NICA diffraction spectra, photographs of the sensors after equilibration and the dependence of the diffraction red shift on the TNT concentration.

The diffraction red shift increased with the TNT concentration. The diffraction red shifted $48 \mathrm{~nm}$ from $533 \mathrm{~nm}$ to $581 \mathrm{~nm}$ (9\% increase) for $5 \mathrm{mM}$ TNT, with the color changing from green to yellow green. The redshift was $60 \mathrm{~nm}$ (11\%) for $10 \mathrm{mM}$ TNT. For 20 mM TNT, the MICA color shifted from green through green-yellow, to yellow, to orange and finally to red, for a total shift of $84 \mathrm{~nm}(16 \%)$. The Figure $5 \mathrm{~A}$ inset shows the colors of the MICAs for different TNT concentrations.

In contrast, the NICA films showed less than a $40 \mathrm{~nm}$ red shift (8\%) for a $20 \mathrm{mM}$ TNT concentration. PMMA colloidal array films showed only a $9 \mathrm{~nm}$ redshift ( $2 \%)$.

The MICA diffraction shift in response to a $20 \mathrm{mM}$ TNT solution was visually evident within $3 \mathrm{~min}$ and the diffraction shift saturated within $15 \mathrm{~min}$. The LOD of this MICA sensor to TNT was calculated to be $1.03 \mu \mathrm{g}$, by assuming a minimum instrument detectable diffraction shift of $0.5 \mathrm{~nm}$.

We used DLS to investigate the dependence of the particle diameter to the TNT concentration. For $10 \mathrm{mM}$ TNT the MICs swelled $21 \mathrm{~nm}$ ( 7 \%) from $304 \mathrm{~nm}$ to $325 \mathrm{~nm}$. In contrast, the NICs only swelled $8 \mathrm{~nm}$ (3\%) from $247 \mathrm{~nm}$ to $255 \mathrm{~nm}$; while the PMMA particles only swelled $2 \mathrm{~nm}$ (1\%) from $194 \mathrm{~nm}$ to $196 \mathrm{~nm}$.

Obviously, the MICA diffraction red shifts did not simply result from swelling of the particle; the relative diffraction shifts should be proportional to the particle diameter increased for a close-packed system. In contrast, the MICA redshift was 2-fold larger than the particle diameter increase in response to adsorption of TNT. The increases in the MICA refractive indexes possibly shortened the incident light wavelength within the MICA sensor adding to the measured diffraction red shift.

As evident from Figure 5, the TNT concentration can be roughly estimated from the color of MICA. For example, when the MICA film turned yellow, the concentration of TNT was $\sim 8 \mathrm{mM}$. 

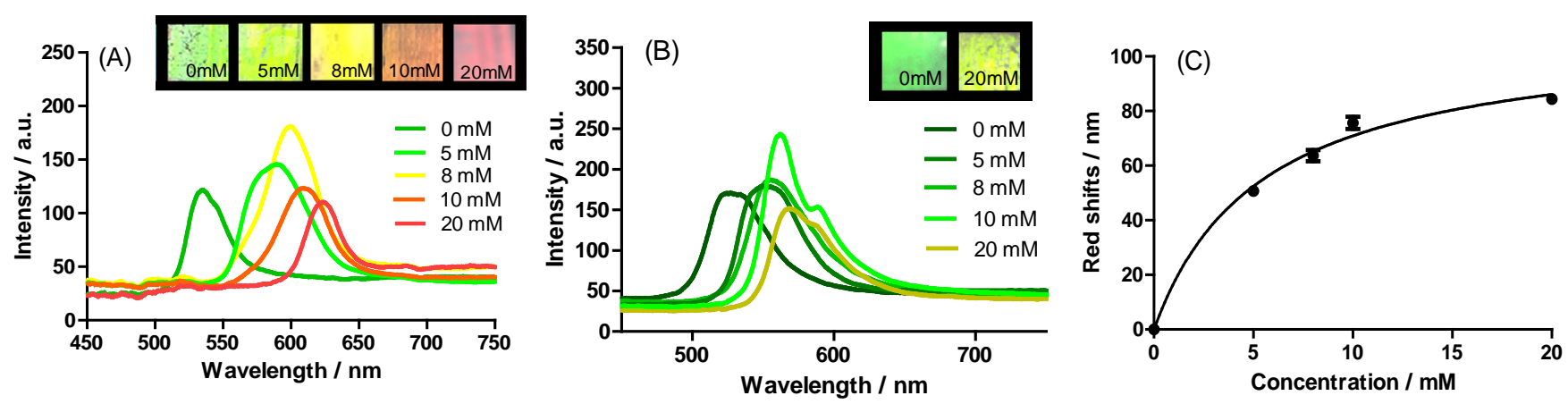

Fig. 5. Diffraction wavelength response and color change of (A) MICA and (B) NICA, and (C) wavelength response curve of TNT on MICs.

To explore the selectivity of the MICA, we incubated the MICA in methanol/water (3/2, v/v) solutions containing $10 \mathrm{mM}$ 2,4-DNT, 2,6-DNT, TNP, 2-nitromesitylene, PNT, ONT, 1,3-dinitrobenzene, methylbenzene, 4-nitrophenol, 2-nitroaniline, 3-aminophenol and 3-nitroaniline, respectively. The responses of MICA to these analogues are shown in Figure 6A. The response of the TNT imprinted MICA to TNT was much higher than that to its analogues. Unlike the MICA, without the target-shaped cavities inside the particles, the analogues of TNT were just able to coat on the surface of NICA, which render a more obvious but similar particle size increasing and red shifts. Only for TNT did the MICA a selectivity compared to the NICA.
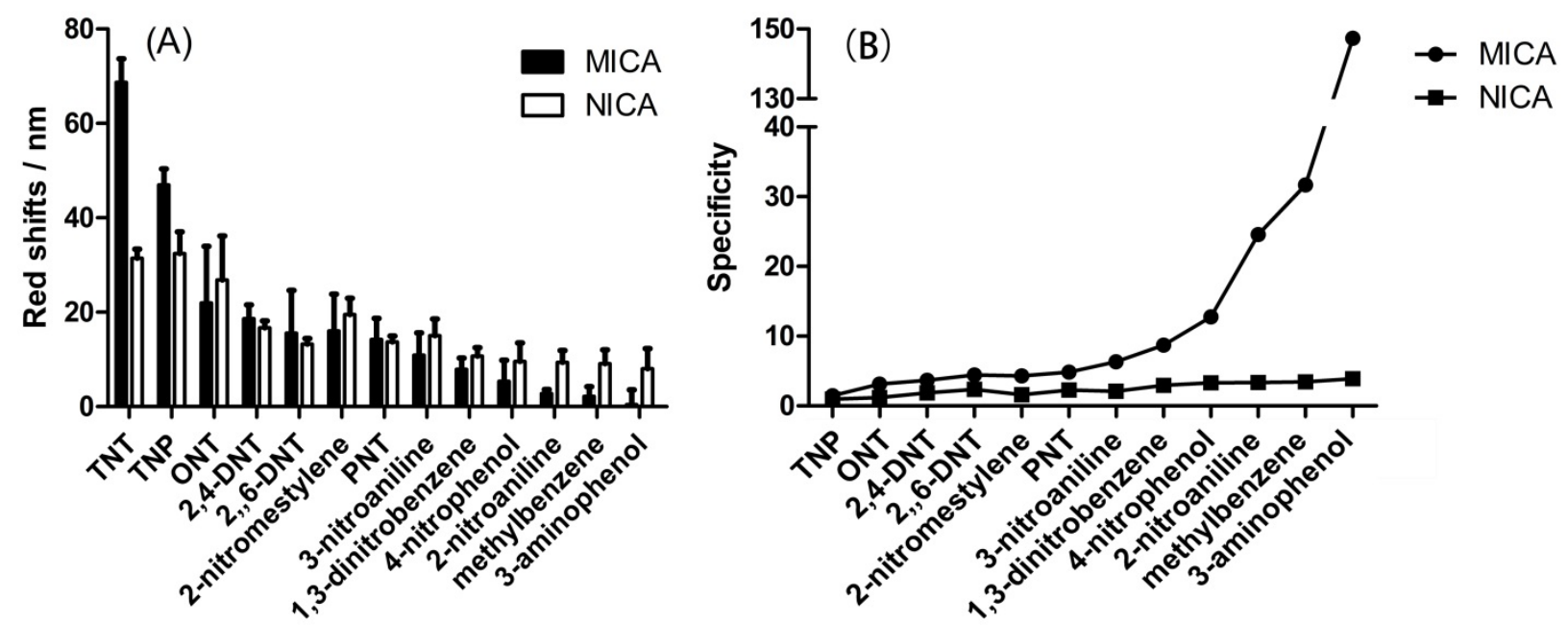

Fig. 6. (A) Histograms of diffraction shifts and (B) specificity of TNT templated MICA response on exposure to TNT and its analogues. 
Figure $6 \mathrm{~B}$ shows the specificity as calculated by the relative diffraction shift response for $10 \mathrm{mM}$ TNT, compared to that for another analogue at $10 \mathrm{mM}$. The specificity increased as the structure of the analogues increasingly differs from that of TNT. The less the structural similarity, the less the analogue bound to the TNT imprinted cavities. Negligible specificity for the NICA was observed.

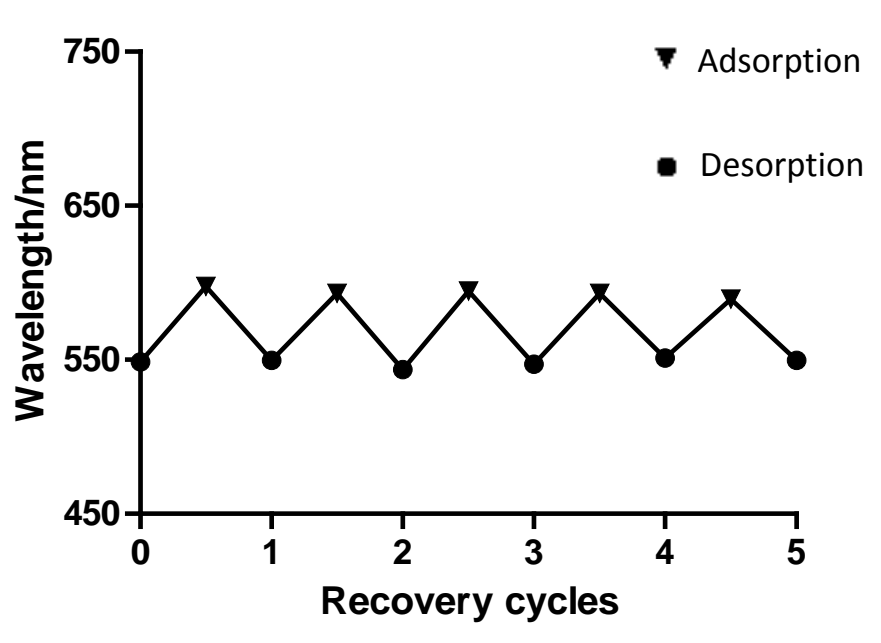

Fig. 7. Reversibility of response of MICA to TNT.

The stability of these MICA films was investigated, which were stored at room temperature without any intentional moisture control for three years. Over this time, less than a $2 \mathrm{~nm}$ diffraction change was observed for sensing $5 \mathrm{mM}$ TNT. The reversibility of MICA diffraction was evaluated by alternatively sensing TNT and then washing off the TNT with $10 \mathrm{~mL}$ methanol/aqueous $(7 / 3, v / v)$ solutions. Figure 7 shows that the MICA diffraction response was reversible over five cycles with a standard deviation of $3 \%$.

Actually, there are lots of meaningful works about TNT sensor based on imprinted materials, and every single method has different emphases, advantages and drawbacks. To evaluate the characteristics of this work and further compare it with other similar detection system, a table was provided as following. 
Table 2

Comparison of similar imprinted detection system for sensing TNT

\begin{tabular}{|c|c|c|c|c|}
\hline Co-technique & Methods & Analyte & Sensor attributes & Ref \\
\hline PC & $\begin{array}{l}\text { a close packed-packed 3D } \\
\text { molecularly imprinted } \\
\text { PMMA colloidal array } \\
\text { which could render the } \\
\text { visual results }\end{array}$ & TNT & $\begin{array}{l}\text { Selectivity: 2,4-DNT, 2,6-DNT, } \\
\text { TNP, 2-nitromesitylene, PNT, } \\
\text { ONT, 1,3-dinitrobenzene, } \\
\text { methylbenzene, 4-nitrophenol, 2- } \\
\text { nitroaniline, 3-aminophenol and 3- } \\
\text { nitroaniline; } \\
\text { LOD: } 1.03 \mu \mathrm{g} ; \\
\text { Reversibility: more than } 5 \text { times; } \\
\text { Stability: more than } 3 \text { years }\end{array}$ & -- \\
\hline QCM & $\begin{array}{l}\text { The potentiodynamic } \\
\text { electro-polymerized MIP } \\
\text { films coated on the Au } \\
\text { quartz crystal resonators }\end{array}$ & $\begin{array}{c}\text { TNP, } \\
\text { TNT, } \\
\text { 2,4-DNT, } \\
\text { 1,3,5-trinitro- } \\
\text { benzene (TNB) }\end{array}$ & $\begin{array}{l}\text { LOD: hundreds (chronoam- } \\
\text { perometry) and tens (piezoelectric } \\
\text { microgravimetry) micromolar }\end{array}$ & [37] \\
\hline $\begin{array}{l}\text { Quantum dots } \\
\text { (QDs) }\end{array}$ & $\begin{array}{l}\text { TNP (dummy template)- } \\
\text { templated imprinted } \\
\text { materials capped with } \\
\text { CdTe quantum dots }\end{array}$ & $\begin{array}{l}\text { TNT } \\
\text { DNT }\end{array}$ & $\begin{array}{l}\text { LOD: } 28 \mu \mathrm{M} \text { to } 40.7 \mu \mathrm{M} \text {; } \\
\text { Selectivity: 2,4-DNP, 4-phenol; } \\
\text { Response time: } 10 \mathrm{~min}\end{array}$ & [25] \\
\hline Fluorescent & $\begin{array}{l}\text { A luminescent cadmium- } \\
\text { pamoate metal-organic } \\
\text { framework prepared under } \\
\text { hydrothermal conditions }\end{array}$ & $\begin{array}{l}\mathrm{TNP}, \\
\mathrm{Cu}^{2+}\end{array}$ & $\begin{array}{l}\text { Selectivity: 4-nitrophenol, 2,6- } \\
\text { DNT, 2,4-DNT, nitrobenzene } \\
\text { (NB), 1,3-dinitrobenzene, } \\
\text { hydroquinone, dimethylbenzene, } \\
\text { bromobenzene } \\
\text { LOD: } 1.76 \times 10^{-8} \mathrm{gL}^{-1} \text {; } \\
\text { Response time: } 2.51 \mathrm{~ns}\end{array}$ & [38] \\
\hline $\begin{array}{l}\text { Unconversion } \\
\text { luminescence } \\
\text { nanoparticles } \\
\text { (UCNPs) }\end{array}$ & $\begin{array}{l}\text { the charge transfer from } \\
\text { UCNPs to TNT by } \\
\text { coating polymer layers } \\
\text { contained amino groups } \\
\text { on the particle surface }\end{array}$ & $\begin{array}{c}\text { TNT (mixture } \\
\text { aqueous solution) }\end{array}$ & $\begin{array}{l}\text { Selectivity: TNP, DNT, NB } \\
\text { LOD: } 9.7 \text { ng/mL }\end{array}$ & [39] \\
\hline
\end{tabular}

\section{Conclusions}

We demonstrated a simple and low cost visual sensing methodology for TNT determination that utilized a self-assembled molecularly imprinted PhC. The TNT imprinted MICA showed selectivity for TNT compared to a broad range of chemical analogues. This MICA sensor was very stable with little response change after three years storage. It visually sensed TNT solutions reversibly. Hopefully, after improving the accuracy and sensitivity, these MICA sensors may also be used to achieve the visual detection results for TNT or other nitro-compounds vapor in an open atmospheric 
place.

\section{Acknowledgements}

The financial support from NSFC (No.U1530141, No. 21375009) and CSC are appreciated S.A.A acknowledges financial support from DTRA (HDTRA1-15-1-0038 HDTRA1-10-1-0044). Scientific advice from Dr. Zhongyu Cai, Mr. Kyle Hufziger, Ms. Natasha Smith and Mr. Andrew Coukouma from Department of Chemistry, University of Pittsburgh are appreciated.

\section{References}

[1] J.S. Caygill, F. Davis, S.P. Higson, Current trends in explosive detection techniques, Talanta, 88 (2012) 14-29.

[2] Z. Meng, Q. Zhang, M. Xue, D. Wang, A. Wang, Removal of 2,4,6-Trinitrotoluene from "Pink Water" Using Molecularly-imprinted absorbent, Propellants Explos. Pyrotech, 37 (2012) 100106.

[3] X. Wang, Z. Mu, F. Shangguan, R. Liu, Y. Pu, L. Yin, Rapid and sensitive suspension array for multiplex detection of organophosphorus pesticides and carbamate pesticides based on silicahydrogel hybrid microbeads, J. Hazard. Mater., 273 (2014) 287-292.

[4] Y. Zhao, J. Wu, J. Huang, Vertical organic nanowire arrays: controlled synthesis and chemical sensors, J. Am. Chem. Soc., 131 (2009) 3158-3159.

[5] H. Tan-Phat, P. Pieta, F. D'Souza, W. Kutner, Molecularly imprinted polymer for recognition of 5-fluorouracil by RNA-type nucleobase pairing, Anal. Chem., 85 (2013) 8304-8312.

[6] J.P. Walker, K.W. Kimble, S.A. Asher, Photonic crystal sensor for organophosphate nerve agents utilizing the organophosphorus hydrolase enzyme, Anal. Bioanal. Chem., 389 (2007) 2115-2124.

[7] H.K. Hunt, M. Armani, Label-free biological and chemical sensors, Nanoscale, 2 (2010) 15441559.

[8] S.D. Alvarez, A.M. Derfus, M.P. Schwartz, S.N. Bhatia, M.J. Sailor, The compatibility of hepatocytes with chemically modified porous silicon with reference to in vitro biosensors, Biomaterials, 30 (2009) 26-34.

[9] E.L. Holthoff, D.N. Stratis-Cullum, M.E. Hankus, A nanosensor for TNT detection based on molecularly imprinted polymers and surface enhanced raman scattering, Sensors, 11 (2011) 2700-2714.

[10] R. Zhang, Y. Wang, L.P. Yu, Specific and ultrasensitive ciprofloxacin detection by responsive photonic crystal sensor, J. Hazard. Mater, 280 (2014) 46-54.

[11] L.D. Bonifacio, D.P. Puzzo, S. Breslav, B.M. Willey, A. McGeer, G.A. Ozin, Towards the photonic nose: a novel platform for molecule and bacteria identification, Adv. Mater., 22 (2010) 1351-1354.

[12] F. Wang, Z. Meng, F. Xue, M. Xue, W. Lu, W. Chen, Q. Wang, Y. Wang, Responsive photonic 
crystal for the sensing of environmental pollutants, Trends in Environ. Anal. Chem., 3-4 (2014) 16.

[13] Q. Fang, J. Geng, B. Liu, D. Gao, F. Li, Z. Wang, G. Guan, Z. Zhang, Inverted opal fluorescent film chemosensor for the detection of explosive nitroaromatic vapors through fluorescence resonance energy transfer, Chem. Eur. J., 15 (2009) 11507-11514.

[14] A. McCluskey, C.I. Holdsworth, M.C. Bowyer, Molecularly imprinted polymers (MIPs): sensing, an explosive new opportunity?, Org. Biomol. Chem., 5 (2007) 3233-3244.

[15] J. Wackerlig, P.A. Lieberzeit, Molecularly imprinted polymer nanoparticles in chemical sensing - Synthesis, characterisation and application, Sens. Actuators, B, 207 (2015) 144-157.

[16] W. Cheong, S. Yang, F. Ali, Molecular imprinted polymers for separation science: A review of reviews, J. Sep. Sci., 36 (2013) 609-628.

[17] L. Chen, S. Xu, J. Li, Recent advances in molecular imprinting technology: current status, challenges and highlighted applications, Chem. Soc. Rev., 40 (2011) 2922-2942.

[18] S. Lordel, F. Chapuis-Hugon, V. Eudes, V. Pichon, Development of imprinted materials for the selective extraction of nitroaromatic explosives, J. Chromatogr.A, 1217 (2010) 6674-6680.

[19] R. Pohle, P. Jeanty, S. Stegmeier, J. Huerttlen, M. Fleischer, Detection of explosives based on the work function read-out of Molecularly Imprinted Polymers, in: R. Walczak, J. Dziuban (Eds.) 26th European Conference on Solid-State Transducers (Eurosensor), Procedia Engineering, Krakow, Poland, 2012, pp. 1370-1373.

[20] J. Saloni, P. Lipkowski, S.S.R. Dasary, Y. Anjaneyulu, H. Yu, G. Hill, Jr., Theoretical study of molecular interactions of TNT, acrylic acid, and ethylene glycol dimethacrylate - elements of molecularly imprinted polymer modeling process, Polymer, 52 (2011) 1206-1216.

[21] B. Johnson-White, M. Zeinali, K.M. Shaffer, C.H. Patterson, Jr., P.T. Charles, M.A. Markowitz, Detection of organics using porphyrin embedded nanoporous organosilicas, Biosens. Bioelectron., 22 (2007) 1154-1162.

[22] R.C. Stringer, S. Gangopadhyay, S.A. Grant, Comparison of molecular imprinted particles prepared using precipitation polymerization in water and chloroform for fluorescent detection of nitroaromatics, Anal. Chim. Acta, 703 (2011) 239-244.

[23] R.C. Stringer, S. Gangopadhyay, S.A. Grant, Detection of nitroaromatic explosives using a fluorescent-labeled imprinted polymer, Anal. Chem., 82 (2010) 4015-4019.

[24] D.C. Apodaca, R.B. Pernites, F.R. Del Mundo, R.C. Advincula, Detection of 2,4-dinitrotoluene (DNT) as a model system for nitroaromatic compounds via molecularly imprinted short-alkylchain SAMs, Langmuir, 27 (2011) 6768-6779.

[25] S. Xu, H. Lu, J. Li, X. Song, A. Wang, L. Chen, S. Han, Dummy molecularly imprinted polymers-capped CdTe quantum dots for the fluorescent sensing of 2,4,6-trinitrotoluene, ACS Appl. Mater. Inter., 5 (2013) 8146-8154.

[26] M. Riskin, R. Tel-Vered, I. Willner, imprinted Au-nanoparticle composites for the ultrasensitive surface plasmon resonance detection of hexahydro-1,3,5-trinitro-1,3,5-triazine (RDX), Adv. Mater., 22 (2010) 1387-1391.

[27] W. Lu, M. Xue, Z. Xu, X. Dong, F. Xue, F. Wang, Q. Wang, Z. Meng, Molecularly imprinted polymers for the sensing of explosives and chemical warfare agents, Curr. Org. Chem., 19 (2015) 62-71.

[28] R.V. Nair, R. Vijaya, Photonic crystal sensors: An overview, Prog. Quant. Electron., 34 (2010) 89-134. 
[29] J. Zhang, L. Wang, X. Chao, S.S. Velankar, S.A. Asher, Vertical spreading of two-dimensional crystalline colloidal arrays, J. Mater. Chem. C, 1 (2013) 6099-6102.

[30] M.M.W. Muscatello, L.E. Stunja, S.A. Asher, Polymerized crystalline colloidal array sensing of high glucose concentrations, Anal. Chem., 81 (2009) 4978-4986.

[31] W. Lu, H. Li, Z. Meng, X. Liang, M. Xue, Q. Wang, X. Dong, Detection of nitrobenzene compounds in surface water by ion mobility spectrometry coupled with molecularly imprinted polymers, J. Hazard. Mater., 280 (2014) 588-594.

[32] F. Xue, Y. Wang, Q. Wang, Z. Meng, M. Xue, S. Huang, W. Lu, Detection of p-nitrophenol using molecularly imprinted colloidal array, Chinese J. Anal. Chem., 40 (2012) 218-223.

[33] W. Lu, F. Xue, S. Huang, Z. Meng, M. Xue, Molecularly imprinted colloidal array for detection of explosives, Chinese J. Anal. Chem., 40 (2012) 1561-1566.

[34] D. Gao, Z. Zhang, M. Wu, C. Xie, G. Guan, D. Wang, A surface functional monomer-directing strategy for highly dense imprinting of TNT at surface of silica nanoparticles, JACS, 129 (2007) 7859-7866.

[35] Equation: One site - specific binding. http://www.graphpad.com/guides/prism/6/curvefitting/index.htm. (Accessed December 29, 2015).

[36] Refractive Index. Info. http://refractiveindex.info/?shelf=other\&book=air\&page=Ciddor. (Accessed December 29, 2015).

[37] H. Tan-Phat, M. Sosnowska, J.W. Sobczak, C.B. Kc, V.N. Nesterov, F. D'Souza, W. Kutner, Simultaneous chronoamperometry and pezoelectric microgravimetry eetermination of nitroaromatic explosives using molecularly imprinted thiophene polymers, Anal. Chem., 85 (2013) 8361-8368.

[38] J. Ye, L. Zhao, R.F. Bogale, Y. Gao, X. Wang, X. Qian, S. Guo, J. Zhao, G. Ning, Highly selective detection of 2,4,6-trinitrophenol and $\mathrm{Cu}^{2+}$ lons based on a fluorescent cadmium-pamoate metal-organic framework, Chem-Eur J., 21 (2015) 2029-2037.

[39] Y. Ma, L. Wang, Upconversion luminescence nanosensor for TNT selective and label-free quantification in the mixture of nitroaromatic explosives, Talanta, 120 (2014) 100-105. 
Graphical abstract: Molecularly imprinted colloidal array (MICA) was explored for the selective visual detection of TNT with color changing from green to red. And molecularly imprinted colloidal particles (MICs) were evaluated for the adsorption capacity and the imprinting efficiency. The MICA had excellent flexibility, reversibility and stability. It promised high potential for the visual semi-quantitative detection of other explosives.

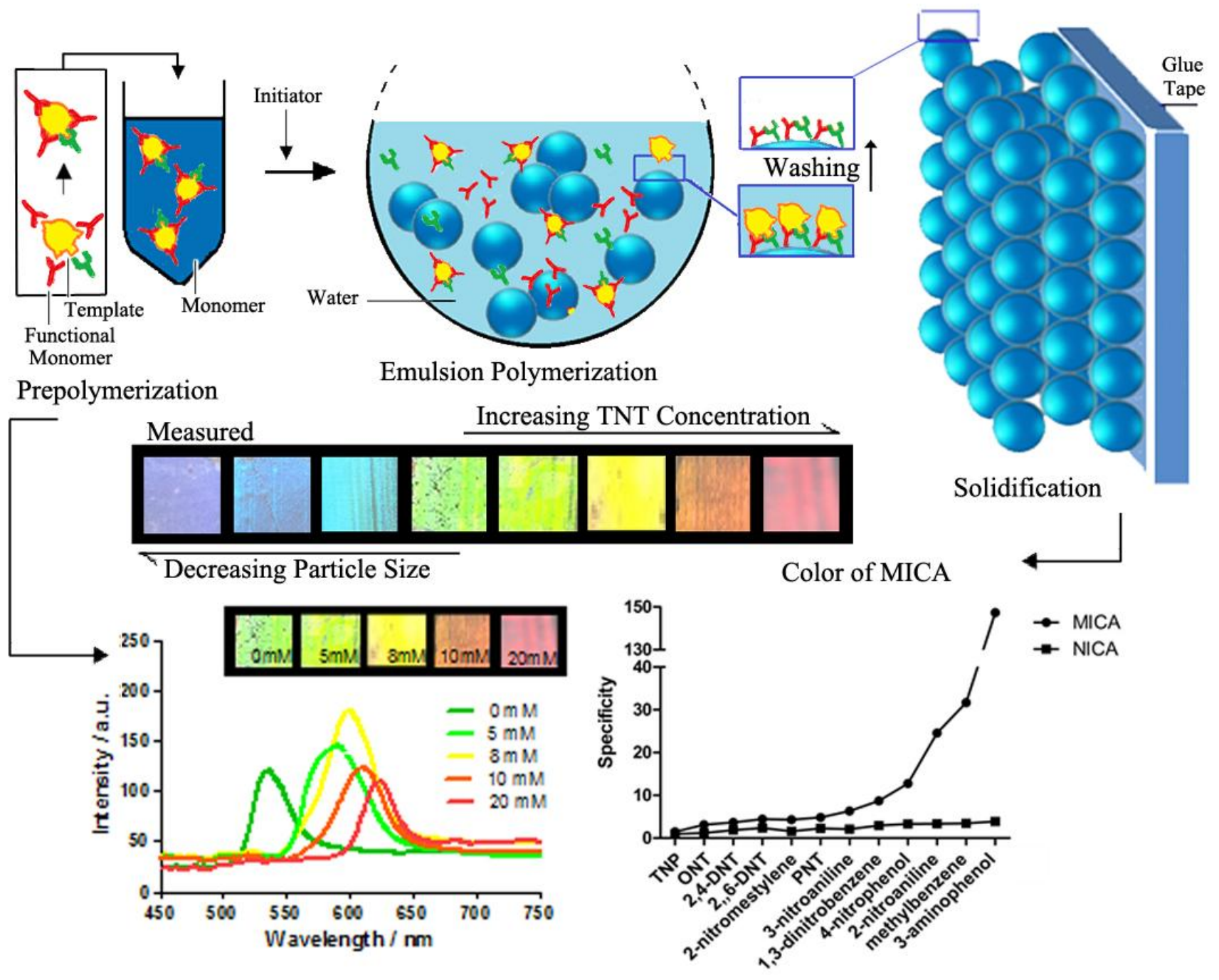

